\title{
Spermidine induces autophagy by inhibiting the acetyltransferase EP300
}

\author{
F Pietrocola ${ }^{1,2,8}$, S Lachkar ${ }^{1,2}$, DP Enot ${ }^{3}$, M Niso-Santano ${ }^{1,2}$, JM Bravo-San Pedro $^{1,2}$, V Sica ${ }^{1,2}$, V Izzo $^{1,2}$, MC Maiuri $^{1,2}$, F Madeo $^{4,5}$, \\ G Mariño* ${ }^{*, 1,2,8}$ and G Kroemer*,1,2,3,6,7
}

Several natural compounds found in health-related food items can inhibit acetyltransferases as they induce autophagy. Here we show that this applies to anacardic acid, curcumin, garcinol and spermidine, all of which reduce the acetylation level of cultured human cells as they induce signs of increased autophagic flux (such as the formation of green fluorescent protein-microtubuleassociated protein 1A/1B-light chain 3 (GFP-LC3) puncta and the depletion of sequestosome-1, p62/SQSTM1) coupled to the inhibition of the mammalian target of rapamycin complex 1 (mTORC1). We performed a screen to identify the acetyltransferases whose depletion would activate autophagy and simultaneously inhibit mTORC1. The knockdown of only two acetyltransferases (among 43 candidates) had such effects: EP300 (E1A-binding protein p300), which is a lysine acetyltranferase, and NAA20 (N( $\alpha)$ acetyltransferase 20, also known as NAT5), which catalyzes the N-terminal acetylation of methionine residues. Subsequent studies validated the capacity of a pharmacological EP300 inhibitor, C646, to induce autophagy in both normal and enucleated cells (cytoplasts), underscoring the capacity of EP300 to repress autophagy by cytoplasmic (non-nuclear) effects. Notably, anacardic acid, curcumin, garcinol and spermidine all inhibited the acetyltransferase activity of recombinant EP300 protein in vitro. Altogether, these results support the idea that EP300 acts as an endogenous repressor of autophagy and that potent autophagy inducers including spermidine de facto act as EP300 inhibitors.

Cell Death and Differentiation (2015) 22, 509-516; doi:10.1038/cdd.2014.215; published online 19 December 2014

Macroautophagy (herein referred to as 'autophagy') consist in the sequestration of cytoplasmic material in autophagosomes, followed by their fusion with lysosomes for the bulk degradation of autophagic cargo by lysosomal hydrolases. ${ }^{1}$ This phenomenon can be measured by following the redistribution of green fluorescent protein-microtubule-associated protein 1A/1B-light chain 3 (GFP-LC3) fusion proteins from a diffuse location to autophagosomes (that results in the formation of the so-called GFP-LC3 'puncta'), the diminution of the overall abundance of autophagic substrates (such as sequestosome1, p62/SQSTM1), and the stereotyped activation of proautophagic signals (such as the inhibition of the mammalian target of rapamycin complex 1, mTORC 1$){ }^{2}$

There is growing consensus that the induction of autophagy by nutritional, pharmacological or genetic interventions can reduce age-related pathologies (such as neurodegenerative diseases or type 2 diabetes) and/or extend longevity. ${ }^{3-6}$ This applies to caloric restriction or intermediate fasting, ${ }^{7}$ continuous or intermittent medication of rapamycin, ${ }^{8-10}$ administration of the sirtuin 1 -activator resveratrol, ${ }^{11,12}$ external supply of the polyamine spermidine, ${ }^{13}$ or genetic ablation of $553 .^{14}$ In all these cases, inhibition of autophagy by deleting or silencing relevant genes abolishes the extension of health span and/or lifespan. ${ }^{13-17}$ Moreover, direct induction of autophagy by transgenic expression of autophagy-relevant genes such as ATG5 in mice is sufficient to increase lifespan. $^{18}$

Recently, acetyltransferases have emerged as a potential target for the pharmaceutical induction of autophagy. Thus, depletion of the sole donor of acetyl groups, acetyl-coenzyme A (acetyl-CoA), is sufficient to reduce the acetylation of cytoplasmic and nuclear proteins coupled to the induction of autophagy. ${ }^{19-22}$ Culture of mammalian cells in nutrient-free (NF) conditions or starvation of mice for $24 \mathrm{~h}$ reduced the intracellular nucleocytosolic concentrations of acetyl-CoA at the same time as autophagy was induced, and replenishment of acetyl-CoA by external sources (for instance, by providing a membrane-permeant precursor of $a$-ketoglutarate for anaplerotic reactions or by microinjection of acetyl-CoA) was sufficient to inhibit starvation-induced autophagy. ${ }^{19-22}$ Beyond the inhibition of acetyltransferases by acetyl-CoA depletion, direct pharmacological inhibition of acetyltransferases might also contribute to the induction of autophagy. A close correlation between autophagy induction and deacetylation of cytoplasmic proteins was observed in a screen conceived to identify autophagy-stimulating polyphenols ${ }^{23}$ as well as in

\footnotetext{
${ }^{1}$ Equipe 11 labellisée par la Ligue Nationale contre le cancer, INSERM U1138, Centre de Recherche des Cordeliers, Paris 75006, France; ${ }^{2}$ Université Paris Descartes, Sorbonne Paris Cité, Paris, France; ${ }^{3}$ Metabolomics and Molecular Cell Biology Platforms, Gustave Roussy, Villejuif 94805, France; ${ }^{4}$ Institute of Molecular Biosciences, University of Graz, Graz 8010, Austria; ${ }^{5}$ BioTechMed Graz, Graz 8010, Austria; ${ }^{6}$ Université de Paris Sud, Villejuif 94805, France and ${ }^{7}$ Pôle de Biologie, Hôpital Européen Georges Pompidou, AP-HP, Paris 75015, France

*Corresponding authors: G Mariño or G Kroemer, Equipe 11 labellisée par la Ligue Nationale contre le cancer, INSERM U1138, Centre de Recherche des Cordeliers, 15 rue de l'ecole de medecine, Paris 75006, France. Tel: +33 1442776 67; Fax: +33 14427 76 74; E-mail: gmg.marino@gmail.com or kroemer@orange.fr

${ }^{8}$ These authors contributed equally to this work.

Abbreviations: EP300; E1A-binding protein p300; GFP, green fluorescent protein; LC3, microtubule-associated protein 1A/1B-light chain 3; mTORC1, mammalian target of rapamycin complex 1; NAA20, $N(\alpha)$-acetyltransferase 20; ATG, autophagy-related; S6RP, S6 ribosomal protein

Received 03.8.14; revised 14.11.14; accepted 19.11.14; Edited by M Piacentini; published online 19.12.14
} 
in vivo experiments designed to explore the health-improving effects of coffee. ${ }^{24}$ Spermidine turned out to be an efficient inhibitor of histone acetyltransferases in vitro ${ }^{13}$ and reduced the global protein acetylation levels in cultured cells. ${ }^{25,26}$

Driven by these premises, we investigated the hypothesis that several health-related compounds including anacardic acid, curcumin, garcinol and spermidine might induce autophagy by inhibition of acetyltranferases. Here we report results supporting this hypothesis. Moreover, we demonstrate that one particular acetyltransferase, EP300 (E1A-binding protein p300), negatively controls autophagy and that anacardic acid, curcumin, garcinol and spermidine may induce autophagy by directly inhibiting EP300.

\section{Results and discussion}

Anacardic acid, curcumin, garcinol and spermidine induce autophagy and deacetylation of cellular proteins. Anacardic acid (6-pentadecyl-salicylic acid from the nutshell of the cashew, Anacardium occidentale), curcumin (from the South Asian spice turmeric, Curcuma longa, one of the principal ingredients of curry powder), garcinol (from the fruit of the Kokum tree, Garcina indica) or spermidine (a polyamine contained in all organisms, but found at particularly high concentrations in some health-related products such as durian fruit, fermented soybeans and wheat germs) were all able to stimulate the formation of GFP-LC3 puncta when added to human U2OS cells stably expressing this fluorescent biosensor (Figures $1 \mathrm{a}$ and $\mathrm{b}$ ). Induction of
GFP-LC3 puncta was also observed in the presence of bafilomycin A1, a specific inhibitor of the vacuolar ATPase required for the fusion between autophagosomes and lysosomes (Figure 1c), supporting the notion that these agents induce autophagic flux. ${ }^{2}$ When added to nontransfected U2OS cells, anacardic acid, curcumin, garcinol and spermidine also stimulated the autophagy-associated lipidation of LC3, which increases its electrophoretic mobility to create the LC3-II form, in both the absence and presence of bafilomycin A1 (Figure 1d), confirming that they induce autophagy. These results were confirmed in additional human cancer cell lines as well as in primary, non-transformed murine embryonic fibroblasts (Supplementary Figure 1). Anacardic acid, curcumin, garcinol and spermidine also reduced the overall lysine acetylation of cellular proteins (Figures $2 a$ and $b$ ), determined by means of a protocol in which cells were fixed in such a way that the plasma membrane but not the nuclear envelope would be permeabilized, allowing a fluorescence-labeled antibody-recognizing proteins with acetylated lysines to gain access to the cytoplasm but not the nucleus. ${ }^{23}$ The magnitude of deacetylation induced by these compounds correlated with their potential to induce autophagy (Figure 2c). Anacardic acid, curcumin, garcinol and spermidine also reduced the phosphorylation of S6RP (S6 ribosomal protein), a substrate of p70S6K (p70S6 kinase), which operates downstream of mTORC1, correlating with their autophagy-inducing capacity (Figures 2d-f). Moreover, all the mentioned acetyltransferase inhibitors induced a significant reduction of p62/SQSTM1 levels, thus confirming (together with the results shown in
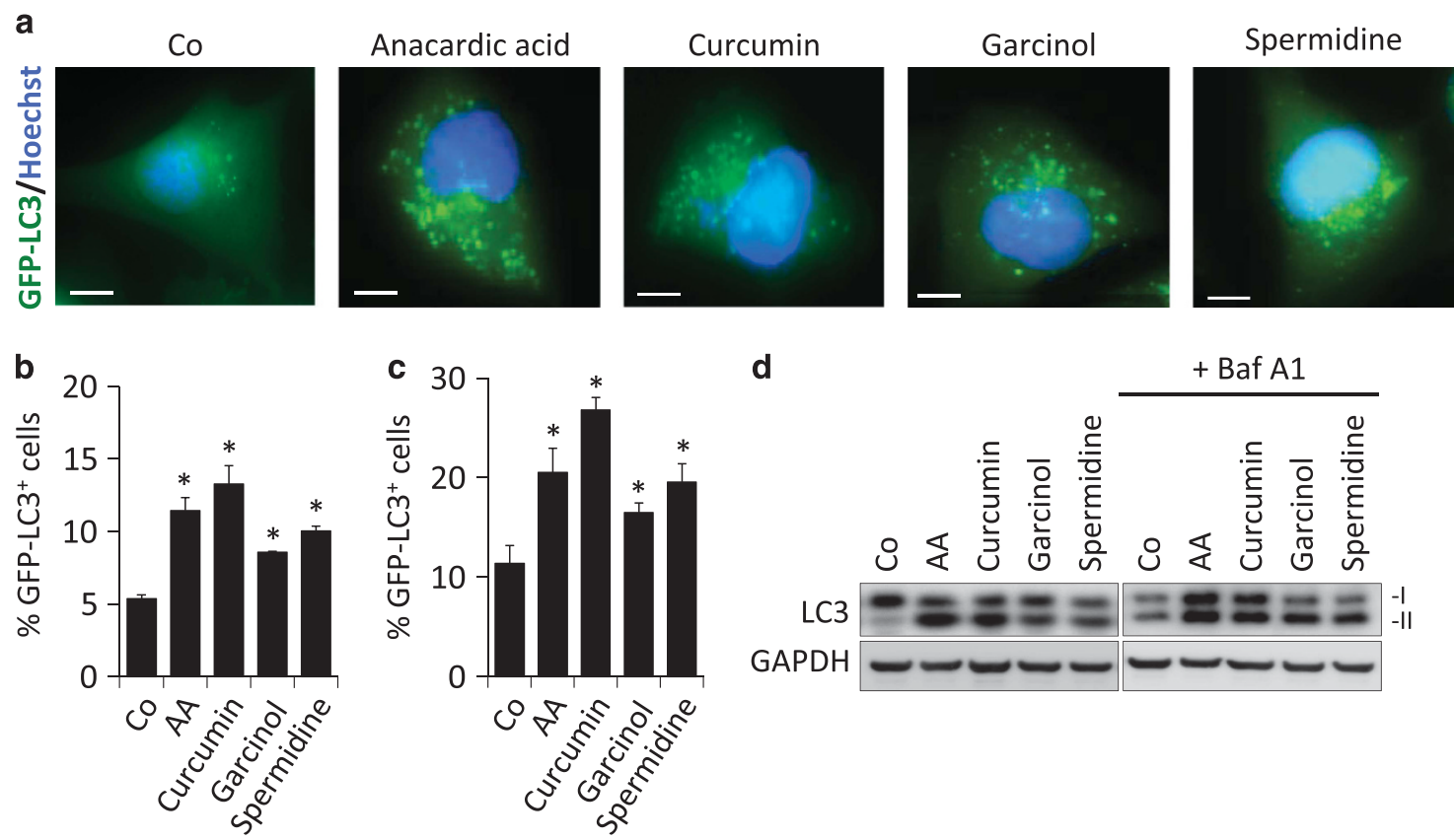

Figure 1 Chemical inhibition of acetyltransferases increases autophagic flux. (a) Representative fluorescence pictures of human U2OS cells stably expressing the autophagosome marker GFP-LC3 after treatment with the indicated acetyltransferase inhibitors for $4 \mathrm{~h}$ in a complete culture media (Co). (b and c) Quantification of GFP-LC3 dots for the data depicted in (a) either in the absence (b) or presence (c) of the lysosomal inhibitor bafilomycin A1 (Baf A1), to measure autophagic flux. (d) Representative immunoblots of total cell lysates showing an increase in LC3-II formation (LC3 lipidation) upon treatment with the indicated acetyltransferase inhibitors either in the presence or absence of Baf A1. Graphic bars represent average and S.E.M. values for at least three independent experiments. ${ }^{*} P$-values $<0.05$ in two-tailed Student's $T$-test as compared with control. Scale bars, $5 \mu \mathrm{m}$ 


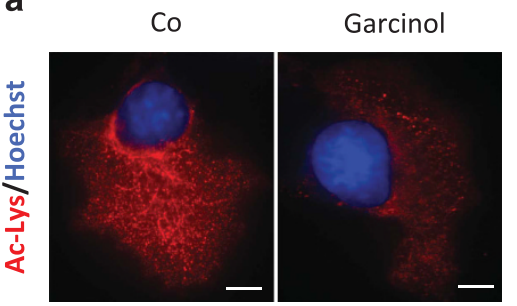

d

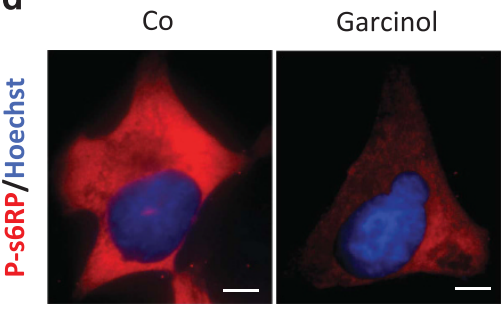

g

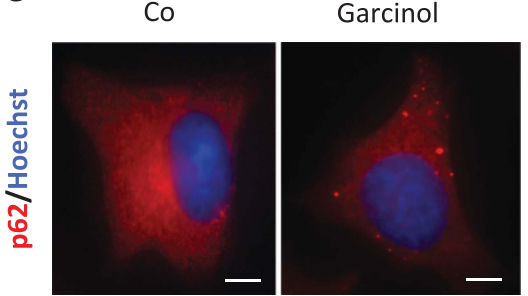

b

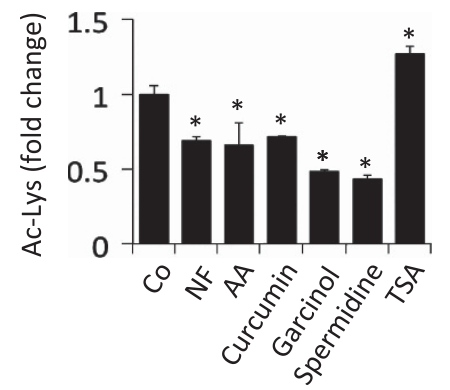

e

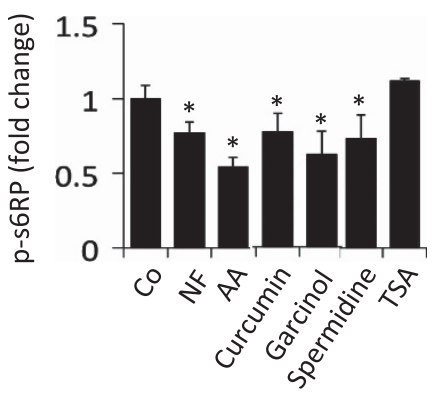

h

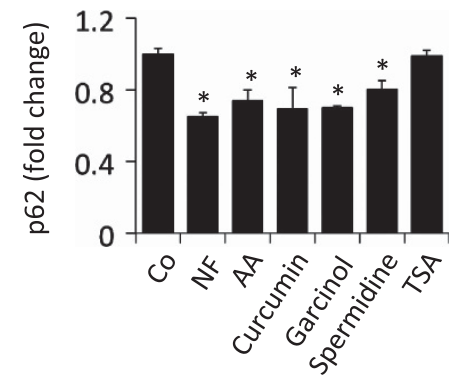

C

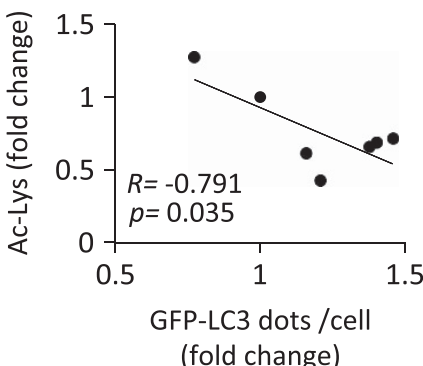

f

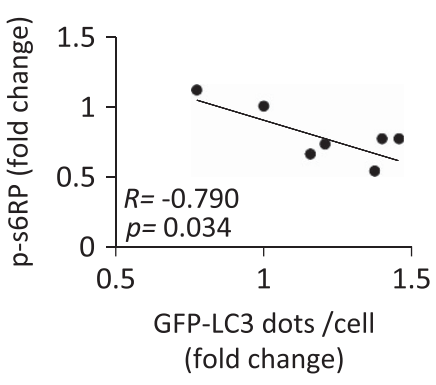

i

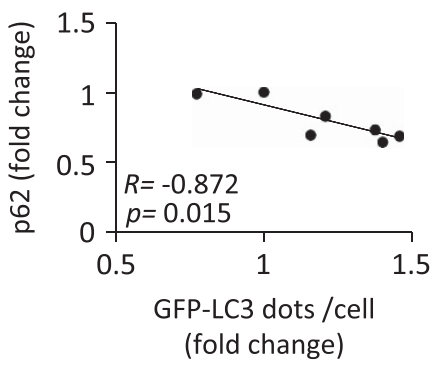

Figure 2 Inhibition of acetyltransferase reduces both cytoplasmic protein acetylation and mTORC1 activity. (a) Representative microphotographs of human U2OS cells either untreated (Co) or treated with acetyltransferase inhibitors (garcinol as an example) after staining for the detection of cytoplasmic acetylated lysine epitopes. (b) Quantification of the average lysine acetylation of cytoplasmic proteins after treatment with different acetyltransferase inhibitors. (c) Scatter graph showing the correlation between the number of GFP-LC3 dots per cell and the average level of cytoplasmic protein lysine acetylation upon treatment with the indicated acetyltransferase inhibitors. NF and trichostatin A (TSA), which were used as controls for decreased (NF) or increased (TSA) cytoplasmic protein acetylation. (d) Representative pictures of U2OS cells treated as in (a) after staining for the detection of phosphorylated ribosomal protein S6 ( $\mathrm{s}-\mathrm{S} 6 \mathrm{RP})$. (e) Quantification of the average phosphorylation level of $p$-S6RP after treatment with acetyltransferase inhibitors. (f) Scatter graph showing the correlation between an increase of GFP-LC3 dots per cell and a decrease of cytoplasmic protein lysine acetylation. (g) Representative pictures of U2OS cells treated as in (a) after staining for the detection of p62/SQSTM1. (h) Quantification of the average cellular level of p62/SQSTM1 after treatment with acetyltransferase inhibitors. (i) Scatter graph showing the correlation between an increase in GFP-LC3 dots per cell and a decrease in p62/SQSTM1 cellular levels. Graphic bars represent average and S.E.M. values for at least three independent experiments. ${ }^{*} P$-values $<0.05$ in two-tailed Student's $T$-test as compared with control. Scale bars, $10 \mu \mathrm{m}$

Figure 1) the induction of a bona fide autophagic flux (Figures $2 \mathrm{~g}$ and $\mathrm{h}$ ). In this regard, we could observe that the reduction of cellular p62/SQSTM1 levels significantly correlated with the increase of GFP-LC3 puncta accumulation (Figure 2i). Altogether, these results reveal the ability of anacardic acid, curcumin, garcinol and spermidine to stimulate a stereotyped molecular cascade of biochemical events that resembles that induced by NF conditions: deacetylation of cellular proteins, inhibition of the mTORC1 pathway and induction of autophagy.

Identification of the acetyltransferase EP300 as a major endogenous repressor of autophagy. To identify the acetyltransferase(s) that control(s) autophagy, we systematically compared the capacity of pharmacological autophagy inducers to the small interfering RNA (siRNA)-mediated knockdown of 43 distinct gene products annotated as protein acetyltransferases with regard to four parameters: (i) induction of GFP-LC3 puncta in the presence of bafilomycin A1, (ii) reduction in the expression of the autophagic substrate p62/SQSTM1 in the absence of bafilomycin A1, (iii) dephosphorylation of s6RP and (iv) protein lysine deacetylation. Knockdown of only two acetyltransferases was able to induce the triad of GFP-LC3 puncta, p62/SQSTM1 depletion, and s6RP dephosphorylation. This applies to EP300, which is a lysine acetyltranferase, and NAA20, (N(a)-acetyltransferase 20, 

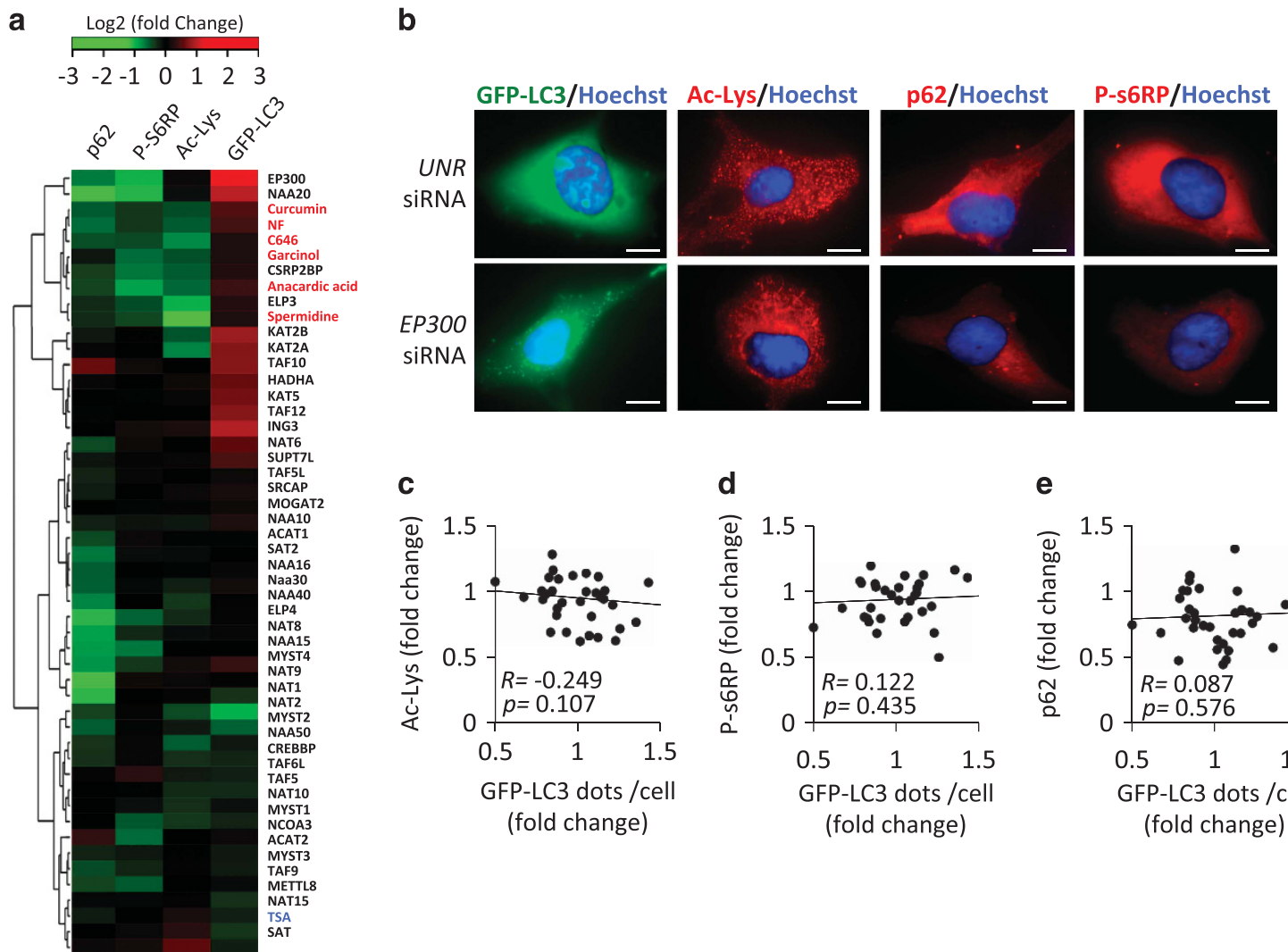

C

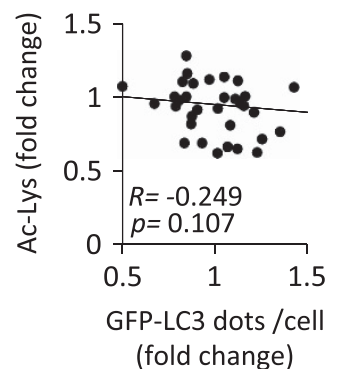

d

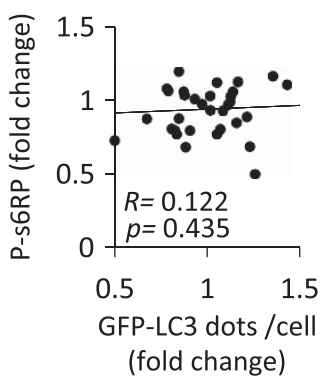

e

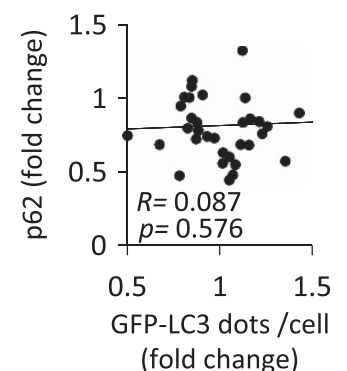

Figure 3 Specific knockdown of a single acetyltransferase has no major impact on the lysine acetylation of cytoplasmic protein. (a) Hierarchical clustering and heat map depicting relative intensity values for immunofluorescence analyses against either p62/SQSTM1, phospho-s6-ribosomal protein (p-s6RP) or acetyl-lysine (Ac-lys) epitopes and GFP-LC3 puncta accumulation in the presence of bafilomycin A1 (Baf A1) after systematic knockdown of 43 distinct acetyltransferases and treatment with different acetyltransferase inhibitors in U2OS cells. In the case of gene knockdown, each square from the heat map represents the average fold change value of two siRNAs targeting each of the acetyltransferase genes. Gene names are depicted in black, whereas chemical acetyltransferase inhibitors and NF conditions are shown in red. Trichostatin A (TSA) used as a positive control for increase in proteins acetylation is shown in blue. (b) Representative pictures of U2OS cells either untreated (Co), treated with acetyltransferase inhibitors (curcumin as an example) or after single knockdown of acetyltransferase genes (EP300 as an example). (c-e) Scatter graph showing the correlation between the number of GFP-LC3 dots per cell and the average level of cytoplasmic protein lysine acetylation (c) or S6RP phosphorylation (d) or p62/SQSTM1 degradation (e) upon specific knockdown of single acetyltransferase genes. Scale bars, $5 \mu \mathrm{m}$

also known as NAT5), which catalyzes the N-terminal acetylation of methionine residues, an irreversible posttranscriptional modification, and not the reversible acetylation of $N$ - $\varepsilon$-lysine groups. The absence of antibodies recognizing acetylated methionine prompted us to focus our studies on the characterization of EP300 activity. Knockdown of EP300 however failed to cause a significant decrease of protein lysine deacetylation (Figures $3 a$ and b). Moreover, we were unable to detect a correlation between overall protein acetylation levels, s6RP phosphorylation or p62/SQSTM1 cellular content and autophagy induction by the knockdown of individual acetyltransferases (Figures $3 c-e$ ). This finding that may reflect the high specificity of such inhibitory manipulations, which target only one single acetyltransferase, compared with the chemical inhibitors, which likely target multiple acetyltransferases. Although knockdown of EP300 failed to reduce the overall acetylation status of cellular proteins (Figures 3a and b), inhibition of EP300 by a 'specific' antagonist, $6646,{ }^{27}$ did cause such an effect if used at a concentration of $10 \mu \mathrm{M}$ (Figure 4a). Careful dose-response studies, however, allowed us to dissociate the capacity of C646 to reduce protein acetylation and to induce GFP-LC3 puncta. At doses ranging from $300 \mathrm{nM}$ to $1 \mu \mathrm{M}, \mathrm{C} 646$ did induce significant levels of LC3B puncta without causing detectable deacetylation of cellular proteins (Figures $4 a$ and b). Thus, the available data indicate that this compound is a more potent autophagy inducer (operating at an $E D_{50}$ of $\sim 1 \mu \mathrm{M})$ than any of the other chemicals used in this study, in line with the fact that C646 can inhibit EP300 at an ED Ef $_{50}$ $\sim 1 \mu \mathrm{M} .^{27}$ Moreover, truly selective inhibition of EP300 (by depletion with two non-overlapping siRNAs or by low-dose C646) could induce autophagy without causing global protein deacetylation. Importantly, C646 potently induced GFP-LC3 puncta with a similar efficiency in intact cells and in cytoplasts, that is, cells that have been enucleated (Figures $5 \mathrm{a}$ and $\mathrm{b}$ ), underscoring the possibility that EP300 mediates autophagy inhibition by cytoplasmic (non-nuclear, transcription-independent) effects. ${ }^{28}$

Spermidine induces autophagy by inhibiting the acetyltransferase activity of EP300. Several of the chemical autophagy inducers used in this study have been described to inhibit EP300. This applies to anacardic acid, ${ }^{29}$ curcumin, $^{30}$ garcinol $^{31}$ and C646. ${ }^{27}$ However, spermidine, 
a

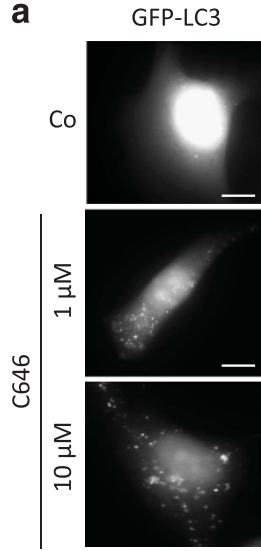

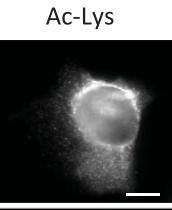

Merged

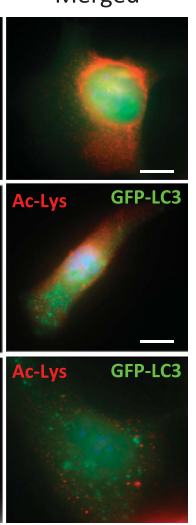

b

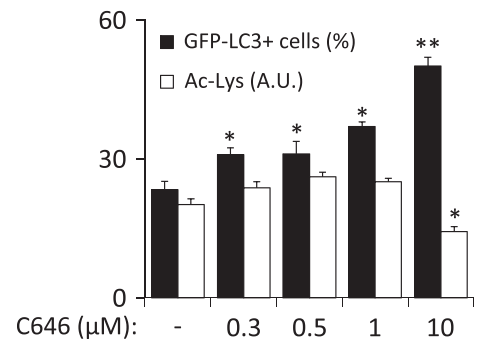

Figure 4 Chemical inhibition of EP300 acetyltransferase increases autophagic flux without affecting general protein acetylation levels. (a) Representative fluorescence pictures of human U2OS cells stably expressing the autophagosome marker GFP-LC3 that were either untreated (Co) or treated with EP300-specific inhibitor C646 at the depicted concentrations after staining for the detection of cytoplasmic acetylated lysine epitopes. (b) Graph showing the percentage of GFP-LC $3^{+}$cells (cells with more than 25 GFP-LC3 puncta) as well as the average lysine acetylation of cytoplasmic proteins after treatment with different concentrations of the EP300 inhibitor C646. Graphic bars represent average and S.E.M. values for at least three independent experiments. ${ }^{*}$-values $<0.05$ in two-tailed Student's $T$-test as compared with control. Scale bars, $5 \mu \mathrm{m}$

a

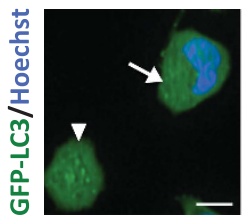

Co

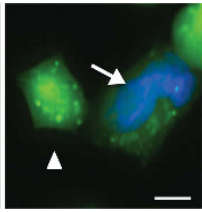

$\mathrm{C} 6461 \mu \mathrm{M}$

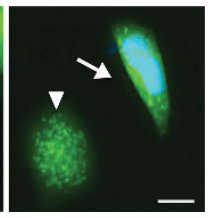

$\mathrm{C} 64610 \mu \mathrm{M}$ b

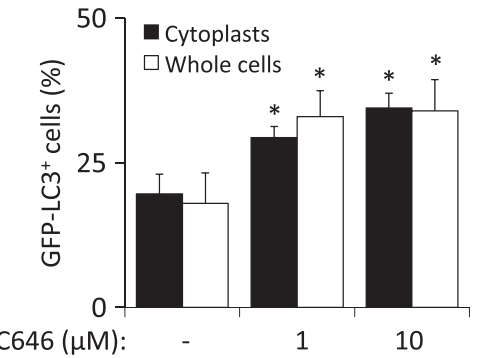

Figure 5 The effects of chemical inhibition of EP300 acetyltransferase are nucleus-independent. (a) Representative pictures for GFP-LC3 puncta accumulation in both enucleated (cytoplasts, arrowheads) and nucleated cells (arrows) treated with C646 at the depicted concentrations. (b) Quantification of the percentage of GFP-LC3 ${ }^{+}$cells or cytoplasts (showing more than 25 GFP-LC3 puncta) after treatment with C646 for $4 \mathrm{~h}$ in the presence of bafilomycin A1 (Baf A1). Graphic bars represent average and S.E.M. values for at least three independent experiments. ${ }^{*} P$-values $<0.05$ in two-tailed Student's $T$-test as compared with control. Scale bars, $5 \mu \mathrm{m}$

which is known to inhibit histone acetyltransferases in yeast (that lacks an EP300 ortholog), has not yet been evaluated for its capacity to interfere with the catalytic activity of EP300. In a cell-free system, spermidine inhibited the capacity of recombinant human EP300 protein to acetylate its substrate histone H3 (Figures $6 \mathrm{a}$ and $\mathrm{b}$ ). This effect was obtained at a physiological concentration of the acetyl donor acetyl-CoA of $10 \mu \mathrm{M}$, yet was attenuated when acetyl-CoA levels were raised 10-fold to $100 \mu \mathrm{M}$ (Figures $6 \mathrm{c}$ and $\mathrm{d}$ and Supplementary Figure 2), suggesting that spermidine acts as a competitive inhibitor, similar to the aforementioned agents. $^{27,29-31}$

Concluding remarks. The results in this paper underscore the notion that inhibitors of acetyltransferases act as potent autophagy inducers. Among the agents evaluated in this work, spermidine may represent the most attractive agent for therapeutic use, for multiple reasons. First, spermidine is a natural compound contained in all cells, including those of our body, which means that all mammals, including humans, physiologically dispose of an endogenous pool of this polyamine. Second, to date no adverse effects of exogenous supply of spermidine have been reported to the best of our knowledge. Third, spermidine has a broad longevityextending activity in multiple species from yeast to rodents. ${ }^{13,32-35}$ Spermidine retards the manifestation of several major age-associated diseases including arterial aging, ${ }^{36}$ colon cancer ${ }^{37}$ and neurodegenerative processes in mice, ${ }^{19,38,39}$ suggesting that it may be used for the prophylaxis of major age-related pathologies.

Here we report the evidence that spermidine may act as an autophagy inducer by virtue of its capacity to inhibit acetyltransferases. Beyond its capacity to reduce the overall levels of protein acetylation, spermidine also inhibits EP300, the acetyltransferase that physiologically acts as a sensor of nutrient-dependent acetyl-CoA levels ${ }^{19}$ and that directly acetylates and inhibits several core autophagy proteins including ATG5, ATG7, ATG12 and LC3. ${ }^{28}$ Thus, the autophagy-repressive activity of EP300 may not only depend on the intracellular concentration of acetyl-CoA but may also be regulated by the presence of spermidine (and likely other polyamines) that acts as a competitive inhibitor or its 

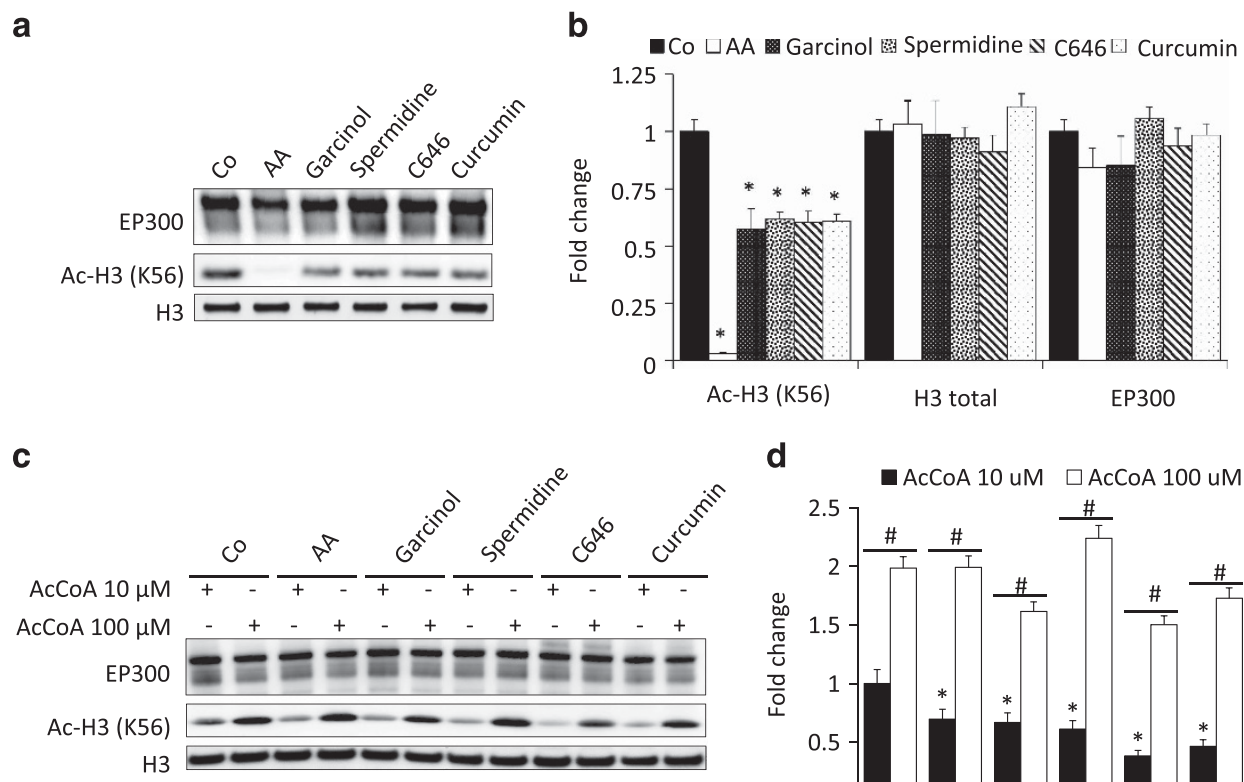

d

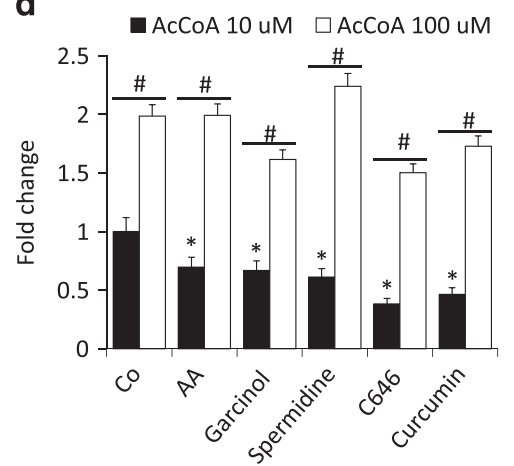

Figure 6 Spermidine competitively inhibits EP300 acetyltransferase activity in a cell-free system. (a) Representative blots showing the inhibition of EP300 in vitro acetyltransferase activity against histone H3, one of EP300 preferred substrates. Anacardic acid (AA) was used as a positive control for EP300 inhibition. (b) Quantification of the data obtained in several independent experiments. (c) EP300 inhibition by different acetyltransferase inhibitors, including spermidine, was reverted by raising the acetyl-CoA $(\mathrm{AcCOA})$ concentration from 10 to $100 \mu \mathrm{M}$, suggesting the competitive nature of EP300 inhibition. (d) Quantification of the data corresponding to several replicate experiments similar to that shown in (c). Graphic bars in (b and $\mathbf{d}$ ) represent average and S.E.M. values for at least three independent experiments. ${ }^{*} P$-value $<0.05$ in two-tailed Student's $T$-test as compared with control in the absence of inhibitors; ${ }^{\#} P$-value $<0.05$ in two-tailed Student's $T$-test as compared with the same condition in the presence of $100 \mu \mathrm{M}$ of $\mathrm{AcCOA}$

acetyltransferase activity. In fact, this is the first insight into the molecular mechanism(s) by which spermidine stimulates autophagic flux in mammalian cells. Further analyses on the capacity of spermidine to inhibit acetyltransferases should elucidate the mechanisms through which this polyamine exerts beneficial effects in diverse pathophysiological contexts. Furthermore, it may be tempting to develop new, truly specific EP300 inhibitors as investigational drugs and perhaps - new lead compounds for the therapeutic induction of autophagy.

\section{Materials and Methods \\ Chemicals, cell lines and culture conditions. Unless otherwise specified, chemicals were purchased from Sigma-Aldrich (St. Louis, MO, USA), culture media and supplements for cell culture were from Gibco-Invitrogen (Carlsbad, CA, USA) and plasticware was from Corning (Corning, NY, USA). Human osteosarcoma U2OS cells and their GFP-LC3-expressing derivatives were cultured in DMEM medium containing $10 \%$ fetal bovine serum, $100 \mathrm{mg} / \mathrm{l}$ sodium pyruvate, $10 \mathrm{mM}$ HEPES buffer, $100 \mathrm{U} / \mathrm{ml}$ penicillin $\mathrm{G}$ sodium and $100 \mathrm{mg} / \mathrm{ml}$ streptomycin sulfate $\left(37^{\circ} \mathrm{C}, 5 \% \mathrm{CO}_{2}\right)$. Cells were seeded in 6- and 12-well plates or in 10 and $15 \mathrm{~cm}$ dishes and grown for $24 \mathrm{~h}$ before treatment with $100 \mu \mathrm{M}$ curcumin, $50 \mu \mathrm{M}$ anacardic acid, $3 \mu \mathrm{M}$ garcinol, 1 or $10 \mu \mathrm{M} \mathrm{C646,} 100 \mu \mathrm{M}$ spermidine and $50 \mu \mathrm{M}$ TSA. For serum and nutrient deprivation, cells were cultured in serum-free Earle's balanced salt solution.}

RNA interference in human cell cultures. siRNAs were reverse transfected with the help of the RNAi MaxTM transfection reagent (Invitrogen, Eugene, OR, USA) according to the manufacturer's instructions. Sequences of the different siRNAs used in this study have been published previously. ${ }^{19}$
Preparation of cytoplasts. U2OS cells stably expressing GFP-LC3 were trypsinized and incubated in $3 \mathrm{ml}$ of complete medium supplemented with $7.5 \mathrm{mg} / \mathrm{ml}$ cytochalasin B for $45 \mathrm{~min}$ at $37^{\circ} \mathrm{C}$. This cell suspension was layered onto a discontinuous Ficoll density gradient $(3 \mathrm{ml}$ of $55 \%, 1 \mathrm{ml}$ of $90 \%$ and $3 \mathrm{ml}$ of $100 \%$ Ficoll-Paques; GE Healthcare, Buckinghamshire, UK) in complete medium containing $7.5 \mathrm{mg} / \mathrm{ml}$ cytochalasin B. Gradients were prepared in ultracentrifuge tubes and pre-equilibrated at $37^{\circ} \mathrm{C}$ in a $\mathrm{CO}_{2}$ incubator overnight. Gradients containing cell suspensions were centrifuged in a prewarmed rotor (SW41; Beckman Coulter, Brea, CA, USA) at $30000 \times g$ for $30 \mathrm{~min}$ at $32^{\circ} \mathrm{C}$. The cytoplastenriched fraction was collected from the interface between 55 and $90 \%$ Ficoll layers, washed in complete medium and incubated for $4 \mathrm{~h}$ at $37^{\circ} \mathrm{C}$ before treatments.

Immunoblotting. For immunoblotting, $25 \mu \mathrm{g}$ of proteins were separated on $4-12 \%$ Bis-Tris acrylamide (Invitrogen) or 12\% Tris-Glycine SDS-PAGE precast gels (Bio-Rad, Hercules, CA, USA) and electrotransferred to Immobilon membranes (Millipore Corporation, Billerica, MA, USA). Membranes were then sliced into different parts according to the molecular weight of the protein of interest to allow simultaneous detection of different antigens within the same experiment. Unspecific binding sites were saturated by incubating membranes for $1 \mathrm{~h}$ in $0.05 \%$ Tween-20 ( $v: v$ in TBS) supplemented with $5 \%$ non-fat powdered milk ( $w: v$ in TBS), followed by an overnight incubation with primary antibody. Development was performed with appropriate horseradish peroxidase (HRP)-labeled secondary antibodies (Southern Biotech, Birmingham, USA) plus the SuperSignal West Pico chemoluminescent substrate (Thermo Scientific-Pierce). An anti-glyceraldehyde-3-phosphate dehydrogenase antibody (Chemicon International, Temecula, CA, USA) was used to control equal loading of lanes.

Automated microscopy. U2OS cells stably expressing GFP-LC3 were seeded in 96-well imaging plates (BD Falcon, Sparks, MD, USA) $24 \mathrm{~h}$ before stimulation. Cells were treated with the indicated agents for $4 \mathrm{~h}$. Subsequently, cells were fixed with 4\% PFA and counterstained with $10 \mu \mathrm{M}$ Hoechst 33342. Images were acquired using a BD pathway 855 automated microscope (BD Imaging 
Systems, San Jose, USA) equipped with a $\times 40$ objective (Olympus, Center Valley, PA, USA) coupled to a robotized Twister II plate handler (Caliper Life Sciences, Hopkinton, MA, USA). Images were analyzed for the presence of GFP-LC3 puncta in the cytoplasm by means of the BD Attovision software (BD Imaging Systems). Cell surfaces were segmented and divided into cytoplasmic and nuclear regions according to standard proceedings. RB $2 \times 2$ and Marr-Hildreth algorithms were used to detect cytoplasmic GFP-LC3-positive dots.

Statistical analyses were conducted using the R software (http://www.r-project.org). For quantitative analyses of protein acetylation, cell surfaces were segmented into cytoplasmic and nucleic regions, and average staining intensity of each individual cell was measured for statistical analysis.

Immunofluorescence. Cells were fixed with $4 \%$ PFA for $15 \mathrm{~min}$ at room temperature and permeabilized with $0.1 \%$ Triton $X-100$ for $10 \mathrm{~min}$, except for staining of cytoplasmic acetyl-lysine-containing proteins, in which any permeabilization step further than PFA fixation was avoided. Nonspecific binding sites were blocked with $5 \%$ bovine serum albumin in PBS, followed by incubation with primary antibodies overnight at $4{ }^{\circ} \mathrm{C}$. Later, the cells were incubated with appropriate Alexa Fluor conjugates (Molecular Probes-Invitrogen, Eugene, OR, USA). In the case of cytoplasmic acetyl-lysine staining, an additional step of blocking using antiacetylated-tubulin antibody $(1: 200)$ was applied. Ten micromoles of Hoechst 33342 (Molecular Probes-Invitrogen) was used for nuclear counterstaining. Fluorescence wide-field and confocal microscopy assessments were performed on an DM IRE2 microscope (Leica Microsystems, Wetzlar, Germany) equipped with a DC300F camera and with an LSM 510 microscope (Carl Zeiss, Jena, Germany), respectively.

Fluorescence microscopy. Confocal fluorescence images were captured using a Leica TCS SPE confocal fluorescence microscope (Leica Microsystems). Non-confocal images were acquired with an Axio Observer inverted fluorescence microscope (Carl Zeiss). For experiments with human cell lines, a Leica APO x63 NA 1.3 immersion objective was used, whereas for the analysis of GFP-LC3 mice tissue sections, a Leica APO x40 NA 1.15 immersion objective was used. Zeiss Immersol immersion oil (Zeiss, Jena, Germany) was used for all microscopic analyses. Images were acquired with a Leica DFC 350 Fx camera (version 1.8.0 using Leica LAS AF software and processed with Adobe Photoshop (version CS2) software (Adobe Systems, San Jose, CA, USA).

In vitro acetylation assay. Recombinant GST-EP300 fusion protein, corresponding to amino acids 1066-1707 (14-418; Millipore, Billerica, MA, USA) was assessed for its acetyltransferase activity on the EP300 natural substrate recombinant histone H3 protein (M2503S; New England Biolabs, Ipswich, MA, USA). Briefly, $1 \mu \mathrm{g}$ of EP300 HAT domain was incubated in the presence of an HAT assay buffer $(250 \mathrm{mM}$ Tris- $\mathrm{HCl}, \mathrm{pH} 8.0,50 \%$ glycerol, $0.5 \mathrm{mM}$ EDTA and $5 \mathrm{mM}$ dithiothreitol), $1 \mu \mathrm{g}$ of histone $\mathrm{H} 3$ protein and 10 or $100 \mu \mathrm{M}$ of acetyl-CoA (A2056; Sigma-Aldrich) for $1 \mathrm{~h}$ at $30^{\circ} \mathrm{C}$. The reaction was stopped by adding $4 \mathrm{x}$ SDS buffer and boiling the samples. Acetylation of substrate proteins was measured by immunoblotting using specific antibodies against H3K56 (Cell Signaling, Danvers, MA, USA).

Statistical analyses. Unless otherwise mentioned, experiments were performed in triplicate and repeated at least two times. Data were analyzed using the GraphPad Prism 5 software (Graphpad software, La Jolla, CA, USA) and statistical significance was assessed by means of two-tailed Student's $t$-test or ANOVA tests, as appropriate.

\section{Conflict of Interest}

The authors declare no conflict of interest.

Acknowledgements. GK is supported by the Ligue contre le Cancer (équipe labelisée); Agence National de la Recherche (ANR); Association pour la recherche sur le cancer (ARC); Cancéropôle lle-de-France; Institut National du Cancer (INCa); Fondation Bettencourt-Schueller; Fondation de France; Fondation pour la Recherche Médicale (FRM); the European Commission (ArtForce); the European Research Council (ERC); the LabEx Immuno-Oncology; the SIRIC Stratified Oncology Cell DNA Repair and Tumor Immune Elimination (SOCRATE); the SIRIC Cancer Research and Personalized Medicine (CARPEM); and the Paris Alliance of Cancer
Research Institutes (PACRI). FM is supported by FWF Grants LIPOTOX, P23490$\mathrm{B} 12, \mathrm{I1000}$ and P24381-B20. MN-S. is supported by the Fondation pour la Recherche Médicale (FRM).

1. Feng Y, He D, Yao Z, Klionsky DJ. The machinery of macroautophagy. Cell Res 2014; 24: 24-41.

2. Klionsky DJ, Abdalla FC, Abeliovich $\mathrm{H}$, Abraham RT, Acevedo-Arozena A, Adeli $\mathrm{K}$ et al. Guidelines for the use and interpretation of assays for monitoring autophagy. Autophagy 2012; 8: 445-544.

3. Levine B, Kroemer G. Autophagy in the pathogenesis of disease. Cell 2008; 132: 27-42.

4. Madeo F, Tavernarakis N, Kroemer G. Can autophagy promote longevity? Nat Cell Biol 2010; 12: 842-846.

5. Rubinsztein DC, Marino G, Kroemer G. Autophagy and aging. Cell 2011; 146: 682-695.

6. Lopez-Otin C, Blasco MA, Partridge L, Serrano M, Kroemer G. The hallmarks of aging. Cell 2013; 153: 1194-1217.

7. Longo VD, Mattson MP. Fasting: molecular mechanisms and clinical applications. Cell Metab 2014; 19: 181-192.

8. Harrison DE, Strong R, Sharp ZD, Nelson JF, Astle CM, Flurkey K et al. Rapamycin fed late in life extends lifespan in genetically heterogeneous mice. Nature 2009; 460: 392-395.

9. Anisimov VN, Zabezhinski MA, Popovich IG, Piskunova TS, Semenchenko AV, Tyndyk ML et al. Rapamycin increases lifespan and inhibits spontaneous tumorigenesis in inbred female mice. Cell cycle 2011; 10: 4230-4236.

10. Blagosklonny MV. Linking calorie restriction to longevity through sirtuins and autophagy: any role for TOR. Cell Death Dis 2010; 1: e12.

11. Hubbard BP, Sinclair DA. Small molecule SIRT1 activators for the treatment of aging and age-related diseases. Trends Pharmacol Sci 2014; 35: 146-154.

12. Sinclair DA, Guarente L. Small-molecule allosteric activators of sirtuins. Annu Rev Pharmacol Toxicol 2014; 54: 363-380.

13. Eisenberg T, Knauer H, Schauer A, Buttner S, Ruckenstuhl C, Carmona-Gutierrez D et al. Induction of autophagy by spermidine promotes longevity. Nat Cell Biol 2009; 11: 1305-1314.

14. Tavernarakis N, Pasparaki A, Tasdemir E, Maiuri MC, Kroemer G. The effects of p53 on whole organism longevity are mediated by autophagy. Autophagy 2008; 4: 870-873.

15. Jia K, Levine B. Autophagy is required for dietary restriction-mediated life span extension in C. elegans. Autophagy 2007; 3: 597-599.

16. Morselli E, Maiuri MC, Markaki M, Megalou E, Pasparaki A, Palikaras K et al. Caloric restriction and resveratrol promote longevity through the Sirtuin-1-dependent induction of autophagy. Cell Death Dis 2010; 1: e10.

17. Bjedov I, Toivonen JM, Kerr F, Slack C, Jacobson J, Foley A et al. Mechanisms of life span extension by rapamycin in the fruit fly Drosophila melanogaster. Cell Metab 2010; 11: 35-46.

18. Pyo JO, Yoo SM, Ahn HH, Nah J, Hong SH, Kam Tl et al. Overexpression of Atg5 in mice activates autophagy and extends lifespan. Nat Commun 2013; 4: 2300.

19. Marino G, Pietrocola F, Eisenberg T, Kong Y, Malik SA, Andryushkova A et al. Regulation of autophagy by cytosolic acetyl-coenzyme A. Mol Cell 2014; 53: 710-725.

20. Schroeder S, Pendl T, Zimmermann A, Eisenberg T, Carmona-Gutierrez D, Ruckenstuhl C et al. Acetyl-coenzyme A: a metabolic master regulator of autophagy and longevity. Autophagy 2014; 10: 1335-1337.

21. Eisenberg T, Schroeder S, Buttner S, Carmona-Gutierrez D, Pendl T, Andryushkova A et al. A histone point mutation that switches on autophagy. Autophagy 2014; 10: 1143-1145.

22. Eisenberg T, Schroeder S, Andryushkova A, Pendl T, Kuttner V, Bhukel A et al. Nucleocytosolic depletion of the energy metabolite acetyl-coenzyme a stimulates autophagy and prolongs lifespan. Cell Metab 2014; 19: 431-444.

23. Pietrocola F, Marino G, Lissa D, Vacchelli E, Malik SA, Niso-Santano M et al. Pro-autophagic polyphenols reduce the acetylation of cytoplasmic proteins. Cell Cycle 2012; 11: 3851-3860

24. Pietrocola F, Malik SA, Marino G, Vacchelli E, Senovilla L, Chaba K et al. Coffee induces autophagy in vivo. Cell Cycle 2014; 13: 1987-1994.

25. Morselli E, Marino G, Bennetzen MV, Eisenberg T, Megalou E, Schroeder S et al. Spermidine and resveratrol induce autophagy by distinct pathways converging on the acetylproteome. J Cell Biol 2011; 192: 615-629.

26. Marino G, Morselli E, Bennetzen MV, Eisenberg T, Megalou E, Schroeder S et al. Longevityrelevant regulation of autophagy at the level of the acetylproteome. Autophagy 2011; 7: 647-649.

27. Bowers EM, Yan G, Mukherjee C, Orry A, Wang L, Holbert MA et al. Virtual ligand screening of the p300/CBP histone acetyltransferase: identification of a selective small molecule inhibitor. Chem Biol 2010; 17: 471-482.

28. Lee IH, Finkel T. Regulation of autophagy by the p300 acetyltransferase. J Biol Chem 2009; 284: $6322-6328$.

29. Devipriya B, Kumaradhas P. Probing the effect of intermolecular interaction and understanding the electrostatic moments of anacardic acid in the active site of p300 enzyme via DFT and charge density analysis. J Mol Graphics Modell 2012; 34: 57-66. 
30. Devipriya B, Kumaradhas P. Molecular flexibility and the electrostatic moments of curcumin and its derivatives in the active site of p300: a theoretical charge density study. Chem-Biol Interact 2013; 204: 153-165.

31. Balasubramanyam K, Altaf M, Varier RA, Swaminathan V, Ravindran A, Sadhale PP et al. Polyisoprenylated benzophenone, garcinol, a natural histone acetyltransferase inhibitor, represses chromatin transcription and alters global gene expression. J Biol Chem 2004; 279 33716-33726.

32. Madeo F, Eisenberg T, Buttner S, Ruckenstuhl C, Kroemer G. Spermidine: a novel autophagy inducer and longevity elixir. Autophagy 2010; 6: 160-162.

33. Minois N, Carmona-Gutierrez D, Bauer MA, Rockenfeller P, Eisenberg T, Brandhorst S et al. Spermidine promotes stress resistance in Drosophila melanogaster through autophagydependent and -independent pathways. Cell Death Dis 2012; 3: e401.

34. Gupta VK, Scheunemann L, Eisenberg T, Mertel S, Bhukel A, Koemans TS et al. Restoring polyamines protects from age-induced memory impairment in an autophagydependent manner. Nat Neurosci 2013; 16: 1453-1460.
35. Kibe R, Kurihara S, Sakai Y, Suzuki H, Ooga T, Sawaki E et al. Upregulation of colonic luminal polyamines produced by intestinal microbiota delays senescence in mice. Scientific Rep 2014; 4: 4548.

36. LaRocca TJ, Gioscia-Ryan RA, Hearon Jr CM, Seals DR. The autophagy enhancer spermidine reverses arterial aging. Mech Ageing Dev 2013; 134: 314-320.

37. Soda K, Kano Y, Chiba F, Koizumi K, Miyaki Y. Increased polyamine intake inhibits ageassociated alteration in global DNA methylation and 1,2-dimethylhydrazine-induced tumorigenesis. PLoS One 2013; 8: e64357.

38. Velloso NA, Dalmolin GD, Gomes GM, Rubin MA, Canas PM, Cunha RA et al. Spermine improves recognition memory deficit in a rodent model of Huntington's disease. Neurobiol Learn Mem 2009; 92: 574-580.

39. Wang IF, Guo BS, Liu YC, Wu CC, Yang CH, Tsai KJ et al. Autophagy activators rescue and alleviate pathogenesis of a mouse model with proteinopathies of the TAR DNA-binding protein 43. Proc Natl Acad Sci USA 2012; 109 15024-15029.

Supplementary Information accompanies this paper on Cell Death and Differentiation website (http://www.nature.com/cdd) 